\title{
Numerical approximation of nonlinear and non-equilibrium two-dimensional model of chromatography
}

\author{
Shamsul Qamar ${ }^{\mathrm{a}, \mathrm{b}, *}$, Sadia Perveen ${ }^{\mathrm{a}}$, Andreas Seidel-Morgenstern ${ }^{\mathrm{b}}$ \\ ${ }^{a}$ Department of Mathematics, COMSATS Institute of Information Technology, \\ Park Road Chak Shahzad Islamabad, Pakistan \\ ${ }^{b}$ Max Planck Institute for Dynamics of Complex Technical Systems, \\ Sandtorstrasse 1, 39106 Magdeburg, Germany
}

\begin{abstract}
This article is concerned with the numerical approximation of a nonlinear model describing the two-dimensional non-equilibrium transport of multi-component mixtures in a chromatographic column. The model consists of nonlinear convection-diffusion partial differential equations coupled with some differential and algebraic equations. Due to the unavailability of analytical solutions for nonlinear models, numerical solution techniques are the only tools to get accurate solutions. A semi-discrete high resolution finite volume scheme is extended to solve the model equations numerically. The scheme is second order accurate in axial and radial coordinates. The accuracy of the scheme is guaranteed by applying a second order accurate Runge-Kutta method to solve the resulting system of ordinary differential equation. The considered radial gradients were typically ignored in pervious studies. They can be relevant in particular in the case of non-perfect injections. The effects of possible rate limitations of the mass transfer in the radial direction are studied assuming hypothetical injections in outer or inner sections of the column inlet cross-section. The case studies consider single-component, two-component and three-component elution. The developed numerical algorithm is an efficient tool to study the effects of mass transfer kinetics on the shape of elution profiles.
\end{abstract}

Keywords: Two-dimensional model of chromatography, non-equilibrium transport,

\footnotetext{
${ }^{*}$ Corresponding author. Tel: +49-391-6110454; fax: +49-391-6110500

Email addresses: qamar@mpi-magdeburg.mpg.de (Shamsul Qamar)
} 
nonlinear adsorption isotherm, mass transfer, finite volume scheme.

\section{Introduction}

Chromatography, originated as a preparative separation technique is one of the greatest methodical phenomenon available to the modern analyst. This technique is successfully employed to target complex separation tasks, which demands high purity of the product for many types of mixtures [1-4]. Application areas of chromatography include (a) purification of the reactant mixtures in chemical synthesis, (b) purification of bio-chemicals such as proteins for bio-pharmaceutical industry, (c) analysis of sample mixtures in forensics, such as body fluid and bio-chemicals, and (d) analysis of environmental samples [1-4].

Chromatographic methods are based on selective adsorption of mixture compounds on a solid phase (or liquid phase) with high surface area. In liquid-solid column chromatography, a mobile (liquid) phase, carrying mixture components is passed through a tabular column containing the stationary (solid) phase. During migration, each component of the mixture interact to varying degrees with the stationary phase. Thus, components of the mixture are continuously partitioned between solid (adsorbent) and mobile phases. Components having strong interaction with the stationary phase propagate slowly along the column compared to the weakly interacting components. A complete separation of the components can be achieved if the column length is long enough [3, 4].

The dynamical behavior of chromatographic columns can be illustrated by several different types of models. These models include the equilibrium dispersive model (EDM), the nonequilibrium lump kinetic model (LKM) and the general rate model (GRM) [1-4]. All models essentially consider nonlinear convection-dominated partial differential equations (PDEs) coupled with some differential or algebraic equations. This article deals with the nonlinear two-dimensional lump kinetic model (2D-LKM) of chromatography. Analytical solutions are possible under linear adsorption conditions only. Therefore, accurate, stable and computationally efficient numerical methods are needed for determining adsorption dynamics inside chromatographic columns $[3,5,6]$. Generally, chromatographic process can be simulated by three well known numerical methods namely, the finite difference, the 
finite element, and the finite volume methods $[3,5,7,8]$. Sharp discontinuities and steep concentration fronts are the fundamental features of nonlinear convection-diffusion partial differential equations. Hence, efficient numerical techniques are needed to capture sharp discontinuities in the profiles at correct positions [3, 5, 9, 10].

The finite volume methods( FVMs) have been widely applied to simulate different chromatographic processes and were found to be a good choice for the numerical simulation of such nonlinear convection dominated problems $[5,6,11,12]$. The FVMs, initially introduced for nonlinear hyperbolic equations, are based upon three main features namely, formal integration, discretization and solution. Such type of schemes are stable and high order accurate on coarse grids. The technique resolve sharp variations by avoiding numerical oscillations and over-predictions in the solutions [9, 10, 13].

This study extends and generalizes our recent study for linear 2D-LKM [14] to nonlinear 2D-LKM. The one-dimensional (1D) high resolution finite volume scheme (HR-FVS) of Koren $[5,13]$ is extended to solve the current 2D-model equations. The scheme is second order accurate in the axial and radial-coordinates. The resulting system of ordinary differential equations (ODEs) is solved by using a second-order Runge-Kutta (RK) method. The considered RK-method is total variation bounded (TVB) which preserves the non-negativity of the solutions in time-coordinate [15]. On the other hand, the local monotonicity in the axial coordinate is guaranteed by the corresponding flux-limiting function [5, 13]. Several challenging case studies of single and multi-component elution are considered to study the effects of axial and radial dispersions on fixed-bed chromatography.

The novelty of this article specifically include: (a) numerical approximation of nonlinear 2D-LKM using the HR-FVS of Koren, (b) injection of specific profiles to amplify the effect of possible rate limitations of the mass transfer in the radial direction, (c) validation of accuracy and efficiency of the proposed numerical scheme through comparison of its results with other flux-limiting schemes $[5,9]$. The develop numerical algorithm and results are seen as helpful tools for further developments of nonlinear chromatographic processes. For instance, the results could be used to study the effects of mass transfer kinetics and axial and radial dispersion coefficients on the elution profiles. The studied 2D-model and 
numerical schemes are more general and flexible than the classical 1D-models and numerical schemes [5]. Further, we have provided useful numerical tools to approximate and apply this model, if required. The latter means if radial dispersion is rate limiting.

The paper is organized as follows. In Section 2, the 2D-LKM is introduced. In Section 3, the proposed HR-FVS is derived. In Section 4, different case studies are considered. Finally, conclusions are drawn in Section 5.

\section{The nonlinear 2D-LKM}

In the case of multi-component nonlinear 2D-LKM, the mass balances for concentrations in the liquid phase are coupled with the kinetic equations for solid phase concentrations. The model assumes that the kinetics of adsorption-desorption are infinitely fast but the mass transfer kinetics are not. The injected solute travels along the column axis in the $z$-direction by advection and axial dispersion, while spreads in the $r$-direction by radial dispersion, see Figure 1. In this study, the flow rate variations are neglected and the interstitial velocity $u$ is kept constant. To trigger and amplify the effect of possible rate limitations of the mass transfer in the radial direction, the following specific injection conditions are assumed. By introducing a parameter $\bar{r}$ the inlet cross-section of the column is divided into an inner cylindrical core and an outer annular ring (c.f. Figure 1). The injection profile is formulated

in a general way allowing for injection either through an inner core, an outer ring or through the whole cross section. The latter case results if $\bar{r}$ is set equal to the radius of the column denoted by $R$.

Under these assumptions, the mass balance equations for concentrations of mixture components in the liquid phase can be expressed as:

$$
\frac{\partial c_{i}}{\partial t}+u \frac{\partial c_{i}}{\partial z}=D_{z, i} \frac{\partial^{2} c_{i}}{\partial z^{2}}+\frac{D_{r, i}}{r} \frac{\partial}{\partial r}\left(r \frac{\partial c_{i}}{\partial r}\right)-\frac{k_{i}}{\epsilon}\left(q_{i}^{*}-q_{i}\right), \quad \text { for } \quad i=1,2, \ldots N_{c} .
$$

The corresponding mass balances for mixture components concentrations in the stationary phase can be expressed as:

$$
\frac{\partial q_{i}}{\partial t}=\frac{k_{i}}{1-\epsilon}\left(q_{i}^{*}-q_{i}\right)
$$


In the above equations $c_{i}(t, z, r)$ and $q_{i}(t, z, r)$ denote the $i$-th component solute concentrations in the liquid and solid phases, respectively. Moreover, $D_{z, i}$ and $D_{r, i}$ represent the longitudinal and radial dispersion coefficients of $i$-th component, $\epsilon \in(0,1)$ is the external porosity, and $N_{c}$ denotes the number of mixture components. In addition, $t, z$ and $r$ denote the time, axial and radial coordinates, respectively. For sufficiently large values of $k_{i}$, for $i=1,2, \ldots N_{c}$, the solution of multi-component 2D-LKM converges to that of multi-component 2D equilibrium dispersive model (2D-EDM).

For modeling and simulation in preparative chromatography, experimentally determined adsorption equilibrium data has to be represented by suitable mathematical equations. Different relations of isotherms are available in the literature [3, 4]. Here, we consider the commonly used nonlinear convex Langmuir isotherm

$$
q_{i}=\frac{a_{i} c_{i}}{1+\sum_{j=1}^{N_{c}} b_{j} c_{j}}, \quad i=1,2,3, \ldots . . N_{c}
$$

where $a_{i}$ represents the Henry's coefficient of $i$-th component and $b_{i}$ quantifies the nonlinearity of the isotherm. To simply the notations and reduce the number of variables appearing in the model equations, the following dimensionless quantities are introduced:

$$
x=\frac{z}{L}, \quad \tau=\frac{u t}{L}, \quad \rho=\frac{r}{R}, \quad P e_{z, i}=\frac{L u}{D_{z, i}}, \quad P e_{r, i}=\frac{R^{2} u}{D_{r, i} L} \quad \kappa_{i}=\frac{k_{i} L}{u},
$$

where $L$ is the height of the column, $P e_{z, i}$ and $P e_{r, i}$ are the dimensionless Peclet numbers in longitudinal and radial-directions, respectively. Using the above dimensionless variables, the model equations in Eqs. (1) and (2) can be rewritten as

$$
\begin{gathered}
\frac{\partial c_{i}}{\partial \tau}=\frac{1}{P e_{z, i}} \frac{\partial^{2} c_{i}}{\partial x^{2}}-\frac{\partial c_{i}}{\partial x}+\frac{1}{P e_{r, i}} \frac{1}{\rho} \frac{\partial}{\partial \rho}\left(\rho \frac{\partial c_{i}}{\partial \rho}\right)-\frac{\kappa_{i}}{\epsilon}\left(q_{i}^{*}-q_{i}\right) \\
\frac{\partial q_{i}}{\partial \tau}=\frac{\kappa_{i}}{(1-\epsilon)}\left(q_{i}^{*}-q_{i}\right)
\end{gathered}
$$

Here, $0 \leq \rho \leq 1$ and $0 \leq x \leq 1$. The model equations represented by Eqs. (5)-(6) are also subjected to the appropriate initial conditions, as well as inlet and outlet boundary 
conditions. The initial conditions for an initially equilibrated column are given as

$$
c_{i}(\tau=0, x, \rho)=c_{i, \text { init }}, \quad q_{i}(\tau=0, x, \rho)=q_{i, \text { init }}^{*}, \quad i=1,2,3, \ldots . . N_{c} .
$$

The corresponding radial boundary conditions (BCs) at $\rho=0$ and $\rho=1$ are expressed as:

$$
\frac{\partial c_{i}(\tau, x, \rho=0)}{\partial \rho}=0, \quad \frac{\partial c_{i}(\tau, x, \rho=1)}{\partial \rho}=0
$$

In this study, the Danckwerts BCs are considered at the column inlet [16]. For the inner zone injection, this boundary condition is expressed as

$$
c_{i}(\rho, x=0, \tau)-\frac{1}{P e_{z, i}} \frac{\partial c_{i}(\rho, x=0, \tau)}{\partial x}= \begin{cases}c_{i, \text { inj }}, & \text { if } 0 \leq \rho \leq \bar{\rho} \text { and } 0 \leq \tau \leq \tau_{\text {inj }} \\ 0, & \bar{\rho}<\rho \leq 1 \text { or } \tau>\tau_{\text {inj }}\end{cases}
$$

while, for the injection through outer annular zone it is given as

$$
c_{i}(\rho, x=0, \tau)-\frac{1}{P e_{z, i}} \frac{\partial c_{i}(\rho, x=0, \tau)}{\partial x}= \begin{cases}c_{i, \mathrm{inj}}, & \text { if } \bar{\rho}<\rho \leq 1 \text { and } 0 \leq \tau \leq \tau_{\mathrm{inj}} \\ 0, & 0 \leq \rho \leq \bar{\rho} \text { or } \tau>\tau_{\mathrm{inj}}\end{cases}
$$

together with the Neumann condition at the outlet of a finite length column

$$
\left.\frac{\partial c_{i}}{\partial x}\right|_{x=1}=0
$$

The symbol $c_{i, \text { inj }}$ represents the concentration of injected $i$-th component, $\tau_{\text {inj }}$ denotes the dimensionless time of injection, and

$$
\bar{\rho}=\bar{r} / R \text {. }
$$

For injection over the whole inlet cross section of the column, either $\bar{\rho}=1$ in Eq. (9) or $\bar{\rho}=0$ in Eq. (10). For $\tau_{\text {inj }}$ greater than the simulation time, the injection becomes continuous.

At the column outlet, the following Neumann BCs are used:

$$
\frac{\partial c_{i}(\tau, x=1, \rho)}{\partial x}=0 .
$$




\section{Numerical Scheme}

Various numerical procedures have been introduced in the literature for approximating the one-dimensional chromatographic models $[3,5]$. In this work, the 1D HR-FVS of Koren $[5,13]$ is extended to solve the current 2D-LKM. The scheme is second order accurate in the axial and radial-coordinates. The resulting system of ODEs is solved by using a second-order Runge-Kutta method. For the sake of simplicity, a single-component 2DLKM is taken into account to derive the numerical scheme. An extension of the scheme to multi-component mixtures is analogous. In this case, Eqs. (5)-(6) for $N_{c}=1$ and $c_{1}:=c$ reduce to

$$
\begin{gathered}
\frac{\partial c}{\partial \tau}=\frac{1}{P e_{z}} \frac{\partial^{2} c}{\partial x^{2}}-\frac{\partial c}{\partial x}+\frac{1}{P e_{r}} \frac{1}{\rho} \frac{\partial}{\partial \rho}\left(\rho \frac{\partial c}{\partial \rho}\right)-\frac{\kappa_{i}}{\epsilon}\left(q^{*}-q\right), \\
\frac{\partial q}{\partial \tau}=\frac{\kappa}{1-\epsilon}\left(q^{*}-q\right) .
\end{gathered}
$$

Here, the HR-FVS is applied to the partial differential equation (PDE) in Eq. (14) only, while the ODE in Eq. (15) is solved by the considered ODE-solver.

The first step in applying the proposed HR-FVS to Eq. (14) is to discretize the computational domain. Let $N_{x}$ and $N_{\rho}$ be the large integers in $x$ and $\rho$-directions, respectively. We assume a Cartesian grid with a rectangular domain $[0,1] \times[0,1]$ which is covered by cells $\Omega_{j l} \equiv\left[x_{j-\frac{1}{2}}, x_{j+\frac{1}{2}}\right] \times\left[\rho_{l-\frac{1}{2}}, \rho_{l+\frac{1}{2}}\right]$ for $1 \leq j \leq N_{x}$ and $1 \leq l \leq N_{\rho}$. The representative coordinates in the cell $\Omega_{j l}$ are denoted by $\left(x_{j}, \rho_{l}\right)$. Here

$$
\left(x_{1 / 2}, \rho_{1 / 2}\right)=(0,0), \quad x_{j}=\frac{x_{j-1 / 2}+x_{j+1 / 2}}{2}, \quad \rho_{l}=\frac{\rho_{l-1 / 2}+\rho_{l+1 / 2}}{2}
$$

and

$$
\Delta x_{j}=x_{j+1 / 2}-x_{j-1 / 2}, \quad \Delta \rho_{l}=\rho_{l+1 / 2}-\rho_{l-1 / 2} .
$$

The cell averaged values of $w_{j, l}(\tau)$ at any time $\tau$ are given as

$$
w_{j, l}=w_{j, l}(\tau)=\frac{1}{\Delta x_{j} \Delta \rho_{l}} \int_{\Omega_{j l}} w(\tau, x, \rho) d x d \rho
$$


Here, $w \in\left\{c, q, q^{*}\right\}$. Till now, the computational data is discretized and the corresponding initial data for $\tau=0$ is allocated to each mesh interval.

Now, integration of Eqs. (14) and (15) over $\Omega_{j l}$ gives

$$
\begin{gathered}
\frac{d c_{j, l}}{d \tau}=-\frac{c_{j+\frac{1}{2}, l}-c_{j-\frac{1}{2}, l}}{\Delta x_{j}}+\frac{1}{\Delta x_{j} P e_{z}}\left[\left(\frac{\partial c}{\partial x}\right)_{j+\frac{1}{2}, l}-\left(\frac{\partial c}{\partial x}\right)_{j-\frac{1}{2}, l}\right] \\
+\frac{1}{\Delta \rho_{l} P e_{r} \rho_{l}}\left[\left(\rho \frac{\partial c}{\partial \rho}\right)_{j, l+\frac{1}{2}}-\left(\rho \frac{\partial c}{\partial \rho}\right)_{j, l-\frac{1}{2}}\right]-\frac{\kappa}{\epsilon}\left(q_{j, l}^{*}-q_{j, l}\right), \\
\frac{d q_{j, l}}{d \tau}=\frac{\kappa}{1-\epsilon}\left(q_{j, l}^{*}-q_{j, l}\right) .
\end{gathered}
$$

Different approximations of the cell interface concentrations along axial-coordinate generate different numerical schemes which are discussed below.

First-order scheme: In this case, the values of concentrations at the cell interfaces in Eq. (19) are approximated as

$$
c_{j+\frac{1}{2}, l}=c_{j, l}, \quad c_{j-\frac{1}{2}, l}=c_{j-1, l} .
$$

This approximation yields a first-order accurate scheme in the axial-direction.

HR-FVS of Koren: The following flux-limiting formula is applied to approximate the cell interface values $c_{j, l+\frac{1}{2}}$ of the concentration in Eq. (19) [13]:

$$
c_{j+\frac{1}{2}, l}=c_{j, l}+\frac{1}{2} \psi\left(\theta_{j+\frac{1}{2}, l}\right)\left(c_{j, l}-c_{j-1, l}\right),
$$

where,

$$
\theta_{j+\frac{1}{2}, l}=\frac{c_{j+1, l}-c_{j, l}+\beta}{c_{j, l}-c_{j-1, l}+\beta},
$$

is the ratio of concentration gradients. Here, division by zero can be avoided by considering $\beta=10^{-10}$. The limiting function $\psi$ is taken as $[13,17]$

$$
\psi\left(\theta_{j+\frac{1}{2}, l}\right)=\max \left(0, \min \left(2 \theta_{j+\frac{1}{2}, l}, \min \left(\frac{1}{3}+\frac{2}{3} \theta_{j+\frac{1}{2}, l}, 2\right)\right)\right) .
$$

Similarly, $c_{j-\frac{1}{2}, l}$ can be calculated by replacing the index $j$ by $j-1$ in the above equations. 
Other flux-limiting schemes: Several other flux-limiting schemes are available in the literature. These schemes are different because each scheme involve different flux-limiting functions $[9,18,19]$. In these schemes, a limited right cell boundary flux is defined as

$$
c_{j+\frac{1}{2}, l}=c_{j, l}+\frac{1}{2} \varphi\left(\eta_{j+\frac{1}{2}, l}\right)\left(c_{j+1, l}-c_{j, l}\right)
$$

The left cell-boundary flux can be approximated analogously. Here, $\eta_{j+\frac{1}{2}, l}$ is given by the following ratio

$$
\eta_{j+\frac{1}{2}, l}=\frac{c_{j, l}-c_{j-1, l}+\beta}{c_{j+1, l}-c_{j, l}+\beta}
$$

In this section, we have selected a few well-known flux-limiters as listed in Table 1. The accuracy and efficiency of the schemes will be analyzed in selected test problems.

Approximations of axial and radial differential terms: The differential values of the concentration at the cell interfaces obtained from the approximation of the axial-dispersion term in Eq. (19) are calculated as

$$
\left(\frac{\partial c}{\partial x}\right)_{j \pm \frac{1}{2}, l}= \pm\left(\frac{c_{j \pm 1, l}-c_{j, l}}{\Delta x_{j}}\right)
$$

Moreover, the differential cell interface values in the radial dispersion term of Eq. (19) are calculated as

$$
\left(\rho \frac{\partial c}{\partial \rho}\right)_{j, l+\frac{1}{2}}=\rho_{l} \max \left(\frac{c_{j, l+1}-c_{j, l}}{\Delta \rho_{l}}, 0\right)+\rho_{l+1} \min \left(\frac{c_{j, l+1}-c_{j, l}}{\Delta \rho_{l}}, 0\right) .
$$

Similarly, the left interface values can be calculated by lowering the index $l$ by one.

Scheme strategy at the boundaries: The flux-limiting formula given by Eqs. (22)(28) are not applicable to the boundary cells. Thus, the first-order scheme is applied to approximate the cell interface values of the concentration in the boundary cells. In the remaining interior cells, one of the above-mentioned second-order accurate HR-FVS scheme can be applied.

ODE-solver: To obtain the second-order accuracy in time, a second-order total variation diminishing (TVD) Runge-Kutta scheme is applied to solve Eqs. (19) and (20). Denoting 
the right-hand side of Eqs. (19) and (20) as $\mathrm{\iota}(w)$ for $w \in\{c, q\}$, a second order TVD Runge-Kutta scheme update $w$ through the following two stages

$$
\begin{aligned}
w^{(1)} & =w^{n}+\Delta \tau \mathrm{E}\left(w^{n}\right), \\
w^{n+1} & =\frac{1}{2}\left(w^{n}+w^{(1)}+\Delta \tau \mathrm{E}\left(w^{(1)}\right)\right),
\end{aligned}
$$

where $w^{n}$ is a solution at previous time step $\tau^{n}$ and $w^{n+1}$ is updated solution at next time step $\tau^{n+1}$. Moreover, $\Delta \tau$ represents the time step which is calculated under the following Courant-Friedrichs-Lewy (CFL) condition

$$
\Delta \tau \leq 0.5 \min \left(\Delta x, \Delta x^{2} P e_{z}, \Delta \rho^{2} P e_{r}, \frac{1-\epsilon}{\kappa}\right)
$$

\section{Numerical case studies}

The proposed numerical scheme is applied to solve the nonlinear 2D-LKM. The accuracy and efficiency of the proposed scheme is analyzed by comparing its results with other fluxlimiting schemes discussed above. In the numerical test problems, one, two, and threecomponent mixtures are considered. In all test problems, the axial and radial dispersion coefficients are assumed to be the same for all mixture components, i.e. $D_{z, i}=D_{z}$ and $D_{r, i}=D_{r}$ for $i=1,2, \cdots, N_{c}$. Moreover, the mass transfer coefficients are also taken the same for all components, i.e. $k_{i}=k$.

\subsection{Single component elution}

In this subsection some test problems of linear and nonlinear single-component elutions are considered.

\section{Case 1: Error analysis of the schemes for linear isotherm.}

The motivation behind this simple study is to analyze the accuracy and efficiency of HRFVS of Koren by comparing its results with other flux-limiting HR-FVS given above. Here, a large radial dispersion coefficient is considered, i.e. $D_{r}=0.1$. Thus, the solution

remains constant along the radial-coordinate, i.e. no radial effects can be observed. Then the resulting $2 \mathrm{D}$ solution is equivalent to the $1 \mathrm{D}$ solution. It is assumed that the column 
is partially pre-loaded in the region $[0.2,0.4] \times[0,1]$ by a sinusoidal profile. The initial conditions are given as

$$
c(0, x, \rho)= \begin{cases}\sin (\pi(x-0.2) / 0.2), & 0.2 \leq x \leq 0.4 \& 0 \leq \rho \leq 1, \\ 0, & \text { otherwise }\end{cases}
$$

Nothing is injected at the column inlet, i.e. $c(t, 0, \rho)=0$, while the zero Neumann BCs is used at the outlet of the column. The column length and radius are $1 \mathrm{~cm}$ and $0.1 \mathrm{~cm}$, $a=1, u=1 \mathrm{~cm} / \mathrm{min}, \epsilon=0.5, k=100 \mathrm{~min}^{-1}$, and the simulation time is $0.6 \mathrm{~min}$. The $L^{1}$-error along the axial-coordinate at $\rho=0$ and at $t_{\max }=0.6 \mathrm{~min}$ is calculated by using the following formula:

$$
L^{1} \text {-error }=\sum_{j=1}^{N_{x}}\left|c_{\text {exact }}\left(t=0.6, x_{j}, \rho=0\right)-c_{\text {Numeric }}\left(t=0.6, x_{j}, \rho=0\right)\right| \Delta x,
$$

where $c_{\text {exact }}$ denotes the exact solution and $c_{\text {Numeric }}$ represents the corresponding numerical solution. Moreover, $N_{x}$ denotes the number of discretization points in the axial-coordinate and $\Delta x$ represents the axial step-size.

Since the radial effects are neglected, the solution has exactly the same value at each point of the radial-coordinate. Further, $k$ is taken very large, e.g. $k=100 \mathrm{~min}^{-1}$. Therefore, the numerical results of the schemes are compared with the following 1D analytical solution which was derived in [13] for the 1D-EDM using the same initial and boundary conditions:

$$
c_{\text {exact }}(t, z, \rho=0)=0.5 \operatorname{real}\left(i e^{p}[\operatorname{erf}(\alpha)-\operatorname{erf}(\beta)]\right),
$$

where, erf represents the error function and

$$
\begin{aligned}
& p=-0.5 D_{\mathrm{z}} t\left(\frac{\pi}{0.2}\right)^{2}+i \frac{\pi}{0.2}(0.2-z-0.5 t), \\
& \alpha=\frac{-0.2+z-0.5 t}{2 \sqrt{0.5 D_{\mathrm{z}} t}}-i \pi \frac{\sqrt{0.5 D_{\mathrm{z}} t}}{0.2} \\
& \beta=\frac{-0.4+z-0.5 t}{2 \sqrt{0.5 D_{\mathrm{z}} t}}-i \pi \frac{\sqrt{0.5 D_{\mathrm{z}} t}}{0.2} .
\end{aligned}
$$

Table 2 presents a comparison of $L^{1}$-errors produced by different numerical schemes. The analysis is done for different values of axial-dispersion coefficients $D_{z}$ using $N_{x} \times N_{r}=$ 
$100 \times 50$ grid points. It can be observed that the Koren scheme gives less errors as compared to the other flux-limiting schemes and has less computational cost. Table 3 displays the $L^{1}$-error and the experimental order of convergence (EOC) of the Koren scheme at different grid points and for different values of $D_{z}$. The EOC of the Koren method is approximately second order (see Table 3) and, thus, seems more effective for such models. The computational cost for the mimod limiter is higher than the other schemes. The backward difference (first order) scheme takes the minimum computational time but produces large errors in the solution. The computational cost of the van-Leer limiter is comparable to the Koren scheme, however it produces large errors. The remaining two limiters have a higher computational costs and lower accuracy. These observations indicate that Koren scheme is a good choices for solving such models.

\section{Case 2: Nonlinear isotherm}

In this case study, we consider the single-component 2D-LKM given by Eqs. (14) and (15) along with the Langmuir nonlinear isotherm $q^{*}(c)=a c /(1+c)$. Both inner and outer zone injections are considered. The Dankwerts boundary conditions given by Eqs. (9), (10) and (13) are applied. All parameters used in this test problems are given in Table 4.

Both inner and outer zone injections are considered. The numerical results at $100 \times 50$ grid points for different values of the dimensionless mass transfer coefficient $\kappa$ are given in Figures 2 and 3 . In the 1D plots for large values of $\kappa$, the typical nonlinear effect of Langmuir isotherm is clearly visible. The effect of radial dispersion coefficient is significantly visible in the 3D-plot of Figures 2 and 3. Moreover, Figure 2 reveals that the solution of 2D-LKM converges to that of 2D-EDM for $\kappa=266.67$.

The $L^{1}$-errors in time at the outlet column $(x=1)$ and at $\rho=0$ can be calculated using the formula

$$
L^{1}-\text { error }=\sum_{n=1}^{N_{T}}\left|c_{R}^{n}-c_{N}^{n}\right| \Delta \tau,
$$

where $c_{R}^{n}:=c_{R}^{n}(\tau, x=1, \rho=0)$ denotes the reference solution for time $\tau^{n}$ and $c_{N}^{n}(\tau, x=$ $1, \rho=0$ ) denotes the corresponding numerical solution. The reference solution was obtained from the Koren scheme at refined mesh, e.g. $N_{x} \times N_{r}=300 \times 100$. Moreover, $N_{T}$ 
denotes the total number of time steps and $\Delta \tau$ represents the time step-size.

Table 5 displays $L_{1}$-errors and CPU times of different numerical schemes for two different values of $\kappa$ at $N_{x} \times N_{r}=100 \times 50$ considering inner zone injection. Once again, Koren scheme is superior as compared to other HR-FVS in term of accuracy and efficiency. For $\kappa=26.67$, the solutions are diffusive, i.e. profiles are smooth. Therefore, errors of all

flux-limiting schemes are equivalent. Figure 4 gives the comparison of solutions obtained from different numerical schemes for $\kappa=26.67$ and $\kappa=266.67$. It can be observed that first order scheme is diffusive, while other schemes have good agreement.

\subsection{Two-component elution with nonlinear isotherm}

After validating the proposed numerical scheme for single-component elution, the test problems of this subsection extend our study to nonlinear two-component elution. In these test problems, two-component mixtures are considered along with nonlinear mixed or standard Langmuir isotherms.

Case 1: Mixed Langmuir isotherm: A mixture of two components is passed through a cylindrical column packed with porous particles. The values of the parameters used in considered simulations are listed in Table 6. Only inner zone injection is considered in this case (c.f. Eq. (9)).

The column is initially equilibrated corresponding to a specific initial composition. A new state is generated by continuously injecting a new composition at the column inlet starting at time $\tau=0$. Such a setup form a specific Riemann problem for negligibly small axial dispersion coefficient. In the solutions, three different elution fronts are usually visible, such as a rarefaction wave (continuous), a shock wave and a semi-shock wave. Shock waves are sharp fronts of the concentration profile. The concentration of components increases across the shock. Thus, shock waves are compression waves. On the other hand, rarefaction waves are expansion waves across which concentration reduces, i.e. are opposite to the compression. The 2D-LKM along with the following generalized Langmuir isotherm given is used to simulate this process $[20,21]$

$$
q_{i}^{*}=a_{i} c_{i} /\left(1+p_{1} b_{1} c_{1}+p_{2} b_{2} c_{2}\right), \quad i=1,2 .
$$


In the current case of mixed Langmuir isotherm, we have chosen $p_{1}=-1$ and $p_{2}=1$. The solutions consist of three states namely, the inlet state on the left $(\mathrm{A})$, the intermediate state at middle (Int), and the initial state on the right (B), separated by two transition waves that can be simple waves or shock waves. Moreover, following abbreviation are used in plots. $\mathrm{S}_{\mathrm{R}}:=$ right shock wave, $\mathrm{S}_{\mathrm{L}}:=$ left shock wave, $\operatorname{Ref}_{\mathrm{R}}:=$ right rarefaction wave and $\operatorname{Ref}_{L}:=$ left rarefaction wave.

Simulation 1 of Case 1: The injected concentrations of components 1 and 2 are taken as, $c_{1, \text { inj }}=0.0451 \mathrm{~g} / \mathrm{l}$ and $c_{2, \mathrm{inj}}=0.0476 \mathrm{~g} / \mathrm{l}$, respectively. The constant initial concentrations of components 1 and 2 are $c_{1, \text { init }}=0.1818 \mathrm{~g} / \mathrm{l}$ and $c_{2 \text {,init }}=0.1142 \mathrm{~g} / \mathrm{l}$. The numerical results at the column outlet $x=1$ are shown in Figure 5 . The 1D plots at $\rho=0$ in Figure 5 contain two constant states, a right shock wave, an intermediate state, and a left rarefaction wave. For small values of $\kappa$, the solutions are more diffusive as compared to the solutions for large values of $\kappa$. Moreover, the solutions fronts could become sharper when $D_{z} \rightarrow 0$. The effect of radial dispersion coefficient $D_{r}$ is visible in all $3 \mathrm{D}$ plots of concentrations plotted with respect to $\tau$ and $\rho$-coordinates. The elapsed time for $\kappa=10^{3}$ is 240 seconds.

Simulation 2 of Case 1: In the second simulation case study, the following data are used to solve the problem by our proposed numerical scheme.

The injected concentrations of components 1 and 2 are taken as, $c_{1, \text { inj }}=0.1638 \mathrm{~g} / \mathrm{l}$ and $c_{2, \text { inj }}=0.1563 \mathrm{~g} / \mathrm{l}$, respectively. The constant initial concentrations of components 1 and 2 are $c_{1, \text { init }}=0.0423 \mathrm{~g} / \mathrm{l}$ and $c_{2, \text { init }}=0.1005 \mathrm{~g} / \mathrm{l}$. The numerical results at column outlet are shown in Figure 6. The solutions contain two constant states, a right rarefaction wave, an intermediate state, and a left shock wave. The elapsed time for $\kappa=10^{3}$ is 250 seconds.

Simulation 3 of Case 1: The data used in the third simulation case study is as follows. The injected concentrations of components 1 and 2 are taken as, $c_{1, \text { inj }}=0.1778 \mathrm{~g} / \mathrm{l}$ and $c_{2, \text { inj }}=0.0667 \mathrm{~g} / \mathrm{l}$, respectively. The constant initial concentrations of components 1 and 2 are $c_{1, \text { init }}=0.0338 \mathrm{~g} / \mathrm{l}$ and $c_{2, \text { init }}=0.2627 \mathrm{~g} / \mathrm{l}$. The numerical results at the column outlet are shown in Figure 7. The solutions contain two constant states, a right rarefaction wave, an intermediate state, and a left rarefaction wave. The elapsed time for $\kappa=10^{3}$ is 250 seconds. 
Case 2: Standard Langmuir isotherm: In this case study, two-component 2D-LKM (c.f. Eqs. (1) and (2) with $N_{c}=2$ ) is considered. The standard nonlinear langumir isotherm (c.f. Eqs. (3) or (38) with $p_{1}=p_{2}=1$ ) is considered for finite feed volumes. The parameters used in this test problem are given in Table 7. A finite width rectangular pulse of the liquid mixture is injected for $t_{\text {inj }}=12 \mathrm{~min}$ at the inner core of the cylindrical column. Both inner and outer zone injections are considered (c.f. Eqs. (9) and (10)). The numerical results are shown in Figures 8 and 9 for two different values of $\kappa$. Concentration plots are generated using $100 \times 50$ grid points and the total computational time is 253 seconds. For small value of mass transfer coefficient, the concentration profiles are wider with long tails. Thus, separation of the components is not good. On the other hand, for large value of $\kappa$ the concentration profiles have narrow rectangular shapes and both components are better separated. Moreover, the column has better efficiency for the large value of $\kappa$ and the solutions of both 2D-LKM and 2D-EDM are the same. The effect of radial dispersion coefficient is significantly visible in all 3D plots of outlet concentrations plotted with respect to $\tau$ and $\rho$-coordinates.

Figure 10 demonstrates the effects of nonequal injection concentrations for three different injections considering inner zone injection, i.e. (a) $c_{1, \text { inj }}=4 \quad g / l$ and $c_{2, \text { inj }}=2 g / l$, (b) $c_{1, \text { inj }}=2 \quad g / l$ and $c_{2, \text { inj }}=1 \quad g / l$, (c) $c_{1, \text { inj }}=1 \quad g / l$ and $c_{2, \text { inj }}=0.5 g / l$. Other parameters of this case study are given in Table 8 .

The numerical results for two different values of $\kappa$ are presented. The solution profiles illustrate the well-known fact that strong nonlinearities produce overshoots in the profiles. The accuracy of the proposed scheme is graphically compared in Figure 11 with other finite volume schemes presented above for $\kappa=10^{4}$. The figure shows that errors of the suggested HR-FVS of Koren are lower than the other schemes. It can be further observed that that errors decreases on reducing the mesh size. From the above observations, we conclude that the suggested HR-FVS of Koren-scheme could be a better option for solving such 2D-models. 


\subsection{Three-component elution with nonlinear isotherm}

In Figure. 12, elution profiles of a three-component mixture are shown. The parameters used in the simulation are $L=10 \mathrm{~cm}, R=0.2 \mathrm{~cm}, u=1 \mathrm{~cm} / \mathrm{min}, \epsilon=0.4, a_{1}=1, a_{2}=3$, $a_{3}=7, b_{i}=1(i=1,2,3), D_{z}=0.01 \mathrm{~cm}^{2} / \min , D_{r}=10^{-4} \mathrm{~cm}^{2} / \min$ and $t_{\max }=180 \mathrm{~min}$. A sample pulse of size one (i.e. $c_{i, \text { inj }}=1 \mathrm{~g} / \mathrm{l}$ for $i=1,2,3$ ) was injected for $t_{\text {inj }}=1 \mathrm{~min}$ through the inner cylindrical core. The column was initially empty i.e $c_{i, \text { init }}=0 \mathrm{~g} / \mathrm{l}$. The numerical results are shown in Figure 12 for two different values of the mass transfer coefficient $\kappa$. Here, components with larger adsorption equilibrium constant elute later from the column as compared to the components having smaller values of the adsorption equilibrium constants. Once again, better separation is achieved for large value of $\kappa$. The effect of radial dispersion coefficient is again visible in all 3D plots.

\section{Conclusion}

This article was concerned with the numerical approximation of a two-dimensional nonequilibrium model of chromatography. A high resolution flux-limiting finite volume scheme was extended to solve the model equations. Concentration profiles over nonlinear chromatographic conditions were investigated for a wide range of flux-limiters in terms of accuracy, stability and computational time. The simulation results showed that Koren scheme is most suitable in terms of accuracy and efficiency for solving the current 2D-LKM. The scheme avoids numerical oscillations and over-predictions in the solutions. Several test problems were analyzed. These problems include, single-component, two-component, and three-component elution. The 2D-model and developed numerical algorithm can be valuable in various situations, e.g. if (a) the injection at the column inlet is not perfect, (b) the column is not homogeneously packed, and (c) there are radial temperature gradients which are also connected with radial concentration gradients. All such situations can happen in reality. In most of the scenarios there effects might be minor or even negligible, justifying the 1D-model. However, for their relevance and effects 2D-models are required. With our isothermal model, we could just study situation (a). Situations (b) and (c) are more 
complicated and require further extensions in the model which are currently in progress.

\section{References}

[1] Guiochon, G., (2002). Preparative liquid chromatography. J. Chromatogr. A 965, 129161.

[2] Guiochon, G., Lin, B., (2003). Modeling for preparative chromatography, Academic Press.

[3] Guiochon, G., Felinger, A., Shirazi, D.G., Katti, A.M., (2006). Fundamentals of preparative and nonlinear chromatography, 2nd ed. ELsevier Academic press, New York.

[4] Ruthven, D.M., (1984). Principles of adsorption and adsorption processes, WileyInterscience, New York.

[5] Javeed, S., Qamar, S., Seidel-Morgenstern, A., Warnecke, G., (2011). Efficient and accurate numerical simulation of nonlinear chromatographic processes. J. Comput. Chem. Eng. 35, 2294-2305.

[6] Lieres, E.V., Andersson, J., (2010). A fast and accurate solver for the general rate model of column liquid chromatography. J. Comput. \& Chem. Eng., 34, 1180-1191.

[7] Javeed, S., Qamar, S., Seidel-Morgenstern, A. Warnecke, G., (2011). A discontinuous Galerkin method to solve chromatographic models. J. Chromatogr. A 1218, 7137-7146.

[8] Rouchon, P., Schonauer, M., Valentin, P.,\& Guiochon, G. (1987). Numerical Simulation of Band Propagation in Nonlinear Chromatography. Sep. Sci. Technol., 22, 1793-1833.

[9] Leer, B.V., (1977). Towards ultimate conservative finite difference scheme.4. A new approach to numerical convection. scheme. J. Comput. Phys., 12, 276-299.

[10] LeVeque, R.J., (1992). Numerical methods for conservation laws. Birkhaüser Verlag, Bassel, Germany. 
[11] Cruz, P., Santos, J.C., Magalhs F.D., Mendes, A., (2005). Simulation of separation processes using finite volume method. J. Comput. $\& 6$ Chem. Eng., 30, 83-98.

[12] Webley, P.A., He, J.,(2000) Fast solution-adaptive finite volume method for PSA/VSA cycle simulation; 1 single step simulation. J. Comput. \& Chem. Eng., 23, 1701-1712.

[13] Koren, B., (1993). A robust upwind discretization method for advection, diffusion and source terms. In C. B. Vreugdenhil, B. Koren, editors, Numerical Methods for AdvectionDiffusion Problems, Volume 45 of Notes on Numerical Fluid Mechanics, chapter 5, pages 117-138, Vieweg Verlag, Braunschweig.

[14] Parveen, S., Qamar, S. and Seidel-Morgenstern, A., (2015). Two-dimensional nonequilibrium model of liquid chromatography: Analytical solutions and moment analysis. Chem. Eng. Sci. 122, 64-77.

[15] S. Gottlieb, C.-W. Shu, Total Variation Diminishing Runge-Kutta Schemes, Mathematics of Computation 67 (1998) 73-85.

[16] Danckwerts, P.V., (1953). Continuous flow systems. J. Chem. Eng. Sci. 2, 1-9.

[17] Sweby, P.K. (1984). High resolution schemes using flux limiters for hyperbolic conservation laws. SIAM J. Numer. Anal., 30, 995-1011.

[18] Roe, P.L., (1986). Characteristic-based schemes for the Euler equations. Annual Review of Fluid Mechanics 18, 337-365.

[19] Leer, B.V., (1974). Towards ultimate conservative finite difference scheme.2. Monotonicity and conservation combined in a second-order scheme. J. Comput. Phys., 14, $361-370$.

[20] Mazzotti, M., 2009. Nonclassical composition fronts in nonlinear chromatography: Delta-shock. Ind. Eng. Chem. Res. 48, 7733-7752. 
[21] Mazzotti, M., Tarafderb, A., Cornela, J., Gritti, F., Guiochon, G., (2010). Experimental evidence of a delta-shock in nonlinear chromatography. J. Chromatogr. A, 1217, 2002-2012.

Table 1: Different flux limiters used in (25)

\begin{tabular}{|c|c|}
\hline Flux limiter & Formula \\
\hline van Leer $([19])$ & $\varphi(r)=\frac{|r|+r}{1+|r|}$ \\
Superbee $([18])$ & $\varphi(r)=\max (0, \min (2 r, 1), \min (r, 2))$ \\
Minmod $([18])$ & $\varphi(r)=\max (0, \min (1, r))$ \\
$\operatorname{MC}([9])$ & $\varphi(r)=\max \left(0, \min \left(2 r, \frac{1}{2}(1+r), 2\right)\right)$ \\
\hline
\end{tabular}


Table 2: Single-component elution (Case 1): $L^{1}$-error and CPU times of schemes at $100 \times 50$ grid points.

\begin{tabular}{|c|c|c|c|c|c|}
\hline \multirow[t]{2}{*}{ Limiter } & \multicolumn{4}{|c|}{$L^{1}$-error } & CPU (s) \\
\hline & $D_{\mathrm{z}}=10^{-3}$ & $D_{\mathrm{z}}=10^{-4}$ & $D_{\mathrm{z}}=10^{-5}$ & $D_{\mathrm{z}}=10^{-6}$ & $D_{\mathrm{z}}=10^{-3}$ \\
\hline First order & 0.05041 & 0.06301 & 0.07438 & 0.06236 & 9 \\
\hline Koren & 0.00092 & 0.00381 & 0.00537 & 0.00546 & 14 \\
\hline van Leer & 0.00372 & 0.00652 & 0.00764 & 0.00769 & 15 \\
\hline Superbee & 0.00425 & 0.00468 & 0.00549 & 0.00564 & 16.0 \\
\hline Minmod & 0.00708 & 0.02068 & 0.02064 & 0.02069 & 19.0 \\
\hline $\mathrm{MC}$ & 0.00316 & 0.00308 & 0.00387 & 0.00612 & 15 \\
\hline
\end{tabular}

Table 3: Single-component elution (Case 1): $L^{1}$-error and EOC of the Koren scheme.

\begin{tabular}{|c|ll|ll|ll|}
\hline Mesh points & $D_{z}=10^{-3}$ & & $D_{z}=10^{-4}$ & & $D_{z}=10^{-5}$ & \\
\hline$N_{x} \times N_{\rho}$ & $L^{1}$-error & EOC & $L^{1}$-error & EOC & $L^{1}$-error & EOC \\
\hline $20 \times 50$ & $4.6 \times 10^{-3}$ & & $8.4 \times 10^{-3}$ & & 0.012 & \\
$40 \times 50$ & $1.5 \times 10^{-3}$ & 1.61 & $2.5 \times 10^{-3}$ & 1.75 & $3.7 \times 10^{-3}$ & 1.70 \\
$80 \times 50$ & $4.85 \times 10^{-4}$ & 1.62 & $6.2 \times 10^{-4}$ & 2.0 & $8.6 \times 10^{-4}$ & 2.10 \\
$160 \times 50$ & $1.52 \times 10^{-4}$ & 1.67 & $1.6 \times 10^{-4}$ & 1.95 & $1.9 \times 10^{-4}$ & 2.18 \\
$320 \times 50$ & $4.35 \times 10^{-5}$ & 1.80 & $4.3 \times 10^{-5}$ & 1.90 & $3.8 \times 10^{-5}$ & 2.32 \\
\hline
\end{tabular}


Table 4: Parameters of single-component elution (Case 2: nonlinear isotherm).

\begin{tabular}{|c|c|}
\hline Parameters & values \\
\hline Length of the Column & $L=4 \mathrm{~cm}$ \\
Radius of the Column & $R=0.2 \mathrm{~cm}$ \\
Radius of inner zone & $\bar{r}=0.1414 \mathrm{~cm}$ \\
Porosity & $\epsilon=0.4$ \\
Interstitial velocity & $u=1.5 \mathrm{~cm} / \mathrm{min}$ \\
Axial dispersion coefficient & $D_{z}=0.01 \mathrm{~cm}^{2} / \mathrm{min}$, \\
Radial dispersion coefficient & $D_{\mathrm{r}}=0.001 \mathrm{~cm} / \mathrm{min}$, \\
Injection time & $t_{\text {inj }}=1 \mathrm{~min}$ \\
Total simulation time & $t_{\mathrm{max}}=20 \mathrm{~min}$ \\
Initial concentration & $c_{\text {init }}=0$ \\
Injected concentration & $c_{\text {inj }}=1$ \\
$a=1$ \\
Adsorption equilibrium constant \\
Langmuir isotherm coefficient
\end{tabular}

Table 5: Single-component elution (Case2: nonlinear isotherm): $L^{1}$-errors and CPU times at $100 \times 50$ grid points.

\begin{tabular}{|c|cc|c|}
\hline Limiter & \multicolumn{2}{|c|}{$L^{1}$-error } & CPU $(\mathrm{s})$ \\
\hline & $\kappa=26.67$ & $\kappa=266.67$ & \\
\hline Koren & 0.024 & 0.028 & 22 \\
van Leer & 0.025 & 0.029 & 22 \\
Superbee & 0.026 & 0.031 & 24 \\
MC & 0.026 & 0.029 & 23 \\
Minmod & 0.027 & 0.032 & 22 \\
First order & 0.050 & 0.093 & 17 \\
\hline
\end{tabular}


Table 6: Parameters of nonlinear two-component LKM (Case 1: Mixed Langmuir isotherm).

\begin{tabular}{|c|c|}
\hline Parameters & values \\
\hline Length of the Column & $L=10 \mathrm{~cm}$ \\
Radius of the Column & $R=0.2 \mathrm{~cm}$ \\
Radius of inner zone & $\bar{r}=0.1414 \mathrm{~cm}$ \\
Porosity & $\epsilon=0.5$ \\
Interstitial velocity & $u=1.0 \mathrm{~cm} / \mathrm{min}$ \\
Axial dispersion coefficient & $D_{z}=10^{-6} \mathrm{~cm}^{2} / \mathrm{min}$, \\
Radial dispersion coefficient & $D_{\mathrm{r}}=10^{-5} \mathrm{~cm} / \mathrm{min}$, \\
Injection time & $t_{\mathrm{inj}}=80 \mathrm{~min}$ \\
$t_{\mathrm{max}}=80 \mathrm{~min}$ \\
Total simulation time \\
$a_{1}=1.5$ \\
$a_{2}=3.0$ \\
Adsorption equilibrium constant for component 1 \\
Adsorption equilibrium constant for component 2 \\
Mixed Langmuir isotherm coefficient for component 1 \\
Mixed Langmuir isotherm coefficient for component 2 \\
$b_{1}=1.0$ \\
$b_{2}=1.0$
\end{tabular}


Table 7: Parameters of two-component 2D-LKM (Case 2: first case study).

\begin{tabular}{|c|c|}
\hline Parameters & values \\
\hline Length of the Column & $L=10 \mathrm{~cm}$ \\
\hline Radius of the Column & $R=0.5 \mathrm{~cm}$ \\
\hline Radius of inner zone & $\bar{r}=0.3536 \mathrm{~cm}$ \\
\hline Porosity & $\epsilon=0.4$ \\
\hline Interstitial velocity & $u=1.0 \mathrm{~cm} / \mathrm{min}$ \\
\hline Axial dispersion coefficient & $D_{z}=10^{-4} \mathrm{~cm}^{2} / \mathrm{min}$ \\
\hline Radial dispersion coefficient & $D_{\mathrm{r}}=10^{-3} \mathrm{~cm}^{2} / \mathrm{min}$ \\
\hline Injection time & $t_{\mathrm{inj}}=12 \mathrm{~min}$ \\
\hline Total simulation time & $t_{\max }=100 \mathrm{~min}$ \\
\hline Initial concentrations & $c_{i, \text { init }}=0 g / l(i=1,2)$ \\
\hline Injection concentrations & $c_{i, \mathrm{inj}}=1 \mathrm{~g} / l(i=1,2)$ \\
\hline Adsorption constant for component 1 & $a_{1}=1$ \\
\hline Adsorption constant for component 2 & $a_{2}=2$ \\
\hline Langmuir isotherm coefficient for component 1 & $b_{1}=0.5$ \\
\hline Langmuir isotherm coefficient for component 2 & $b_{2}=1$ \\
\hline
\end{tabular}


Table 8: Parameters of two-component 2D-LKM (Case 2: second case study).

\begin{tabular}{|c|c|}
\hline Parameters & values \\
\hline Length of the Column & $L=1 \mathrm{~cm}$ \\
\hline Radius of the Column & $R=0.2 \mathrm{~cm}$ \\
\hline Radius of inner zone & $\bar{r}=0.1414 \mathrm{~cm}$ \\
\hline Porosity & $\epsilon=0.4$ \\
\hline Interstitial velocity & $u=0.1 \mathrm{~cm} / \mathrm{min}$ \\
\hline Axial dispersion coefficient & $D_{z}=10^{-4} \mathrm{~cm}^{2} / \mathrm{min}$ \\
\hline Radial dispersion coefficient & $D_{\mathrm{r}}=10^{-4} \mathrm{~cm}^{2} / \mathrm{min}$ \\
\hline Injection time & $t_{\mathrm{inj}}=12 \min$ \\
\hline Total simulation time & $t_{\max }=100 \mathrm{~min}$ \\
\hline Initial concentrations & $c_{i, \text { init }}=0 g / l(i=1,2)$ \\
\hline Adsorption constant for component 1 & $a_{1}=1.0$ \\
\hline Adsorption constant for component 2 & $a_{2}=2.0$ \\
\hline Langmuir isotherm coefficient for component 1 & $b_{1}=0.05$ \\
\hline Langmuir isotherm coefficient for component 2 & $b_{2}=0.1$ \\
\hline
\end{tabular}




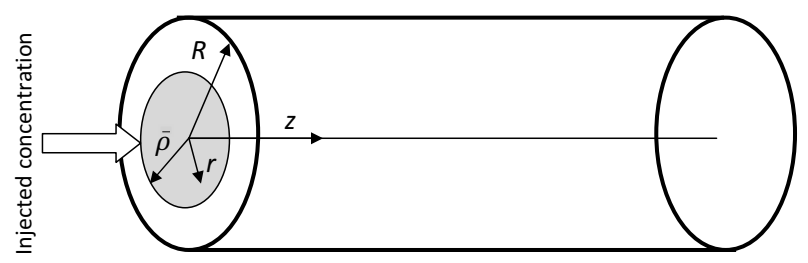

Figure 1: Schematic diagram of a chromatographic column of cylindrical geometry.
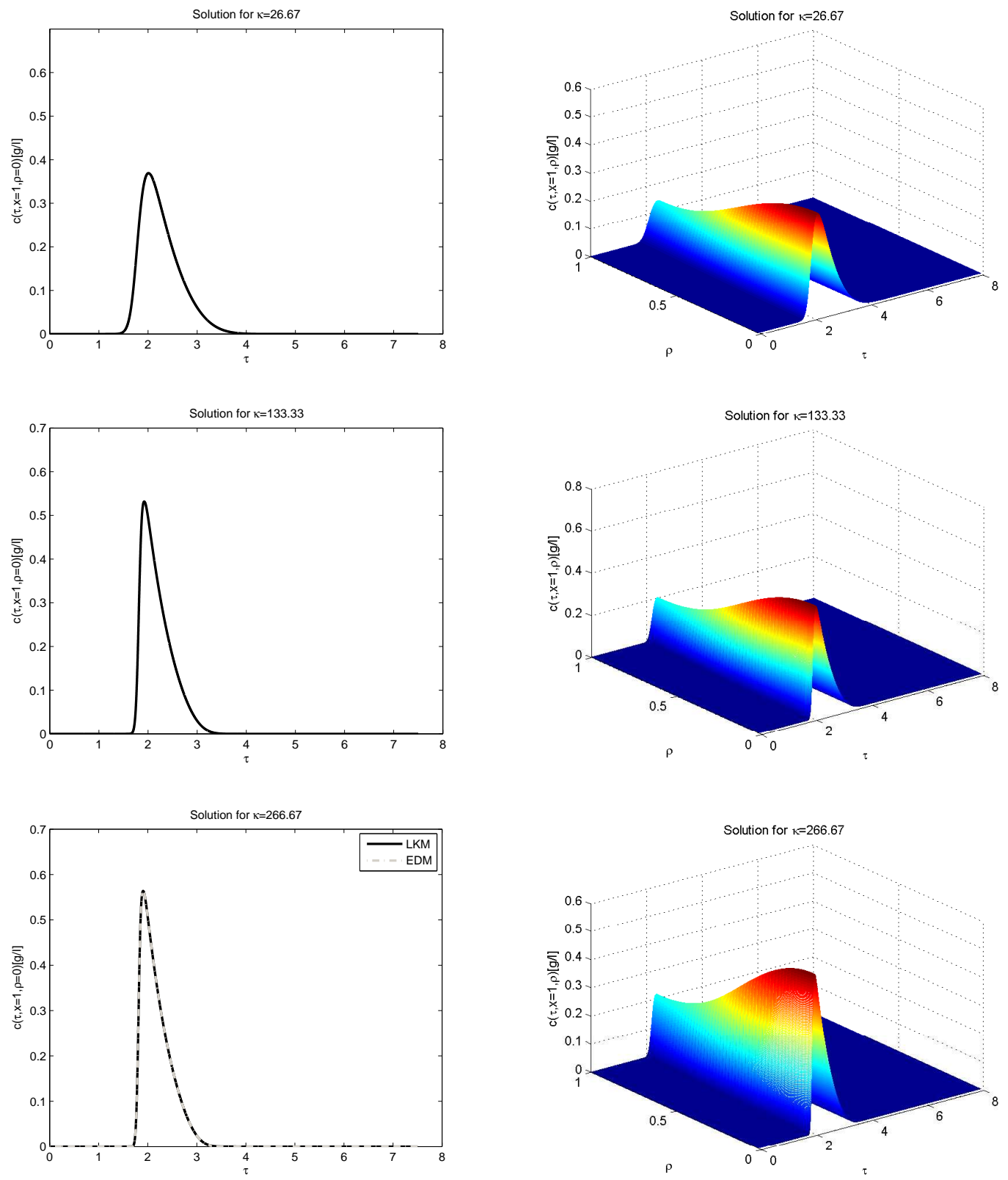

Figure 2: Effect of $\kappa$ on nonlinear single-component elution using $100 \times 50$ grid points and inner zone injecion. 

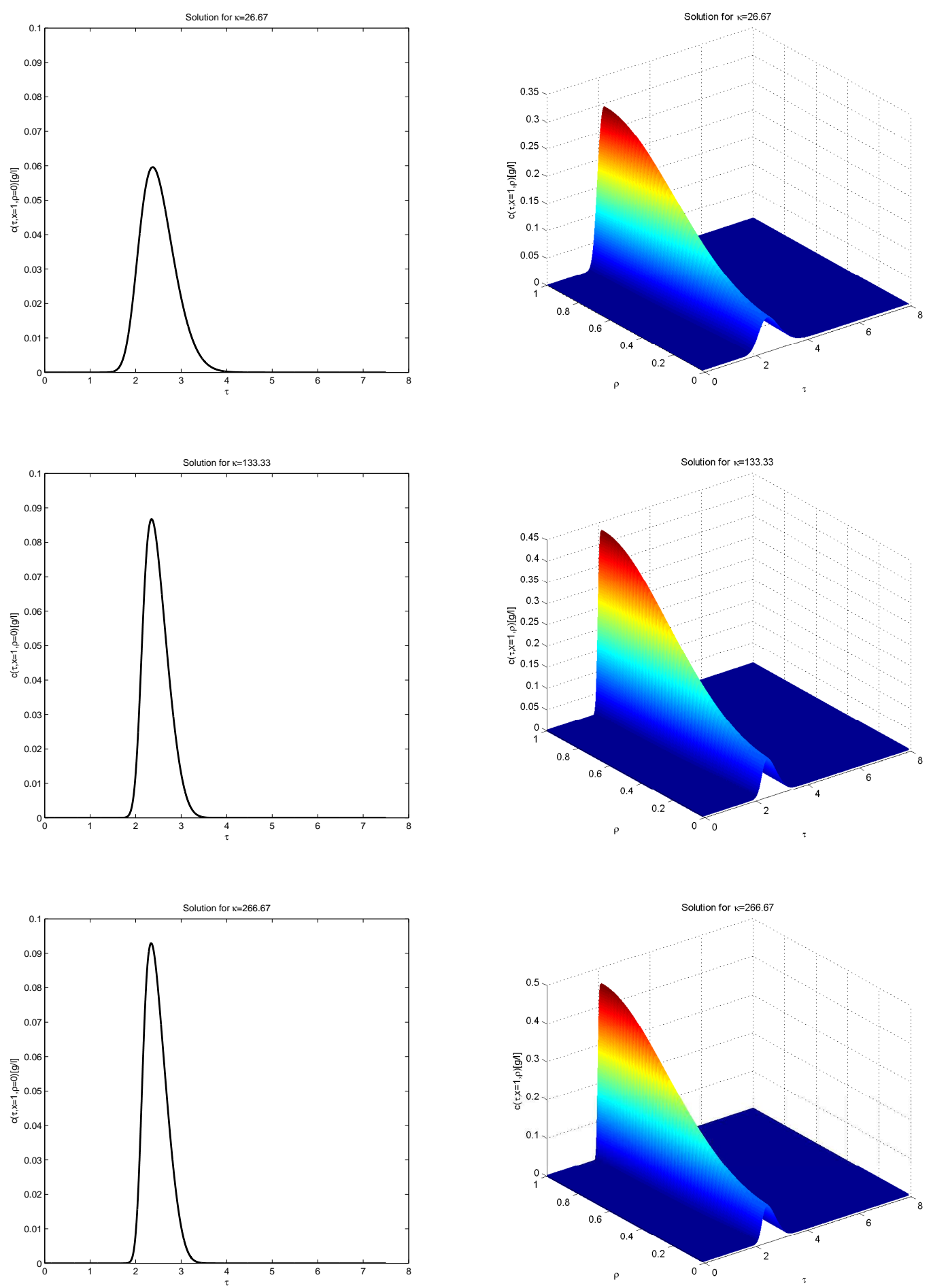

Figure 3: Effect of $\kappa$ on nonlinear single-component elution considering $100 \times 50$ grid points and outer zone injection. 

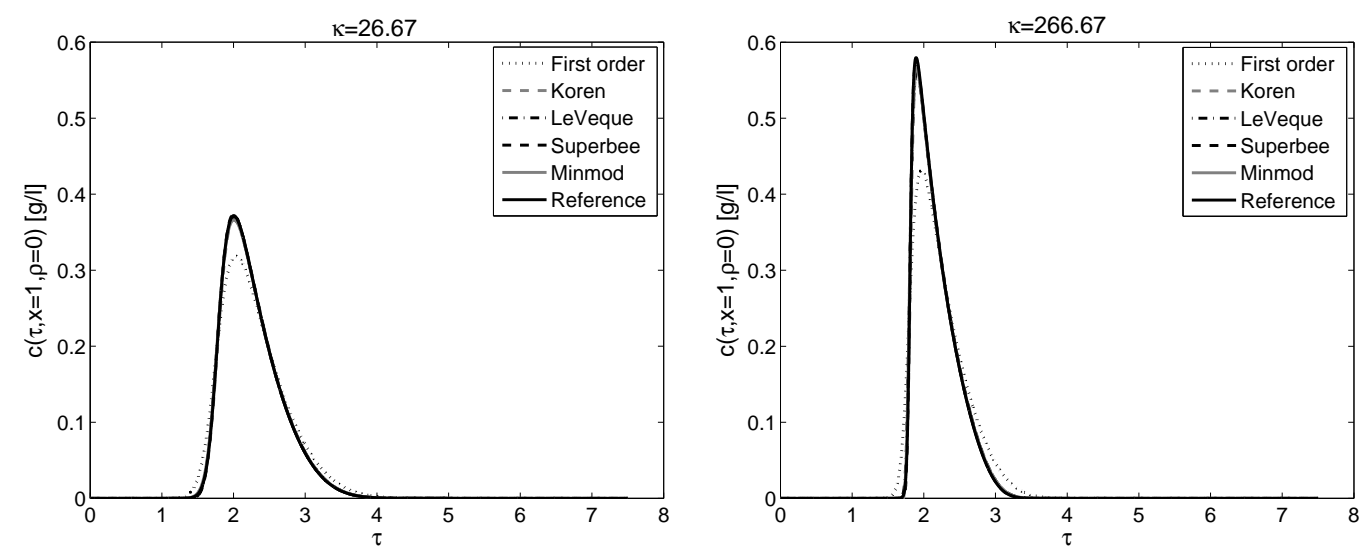

Figure 4: Comparison of schemes for nonlinear single-component elution using $100 \times 50$ grid points.
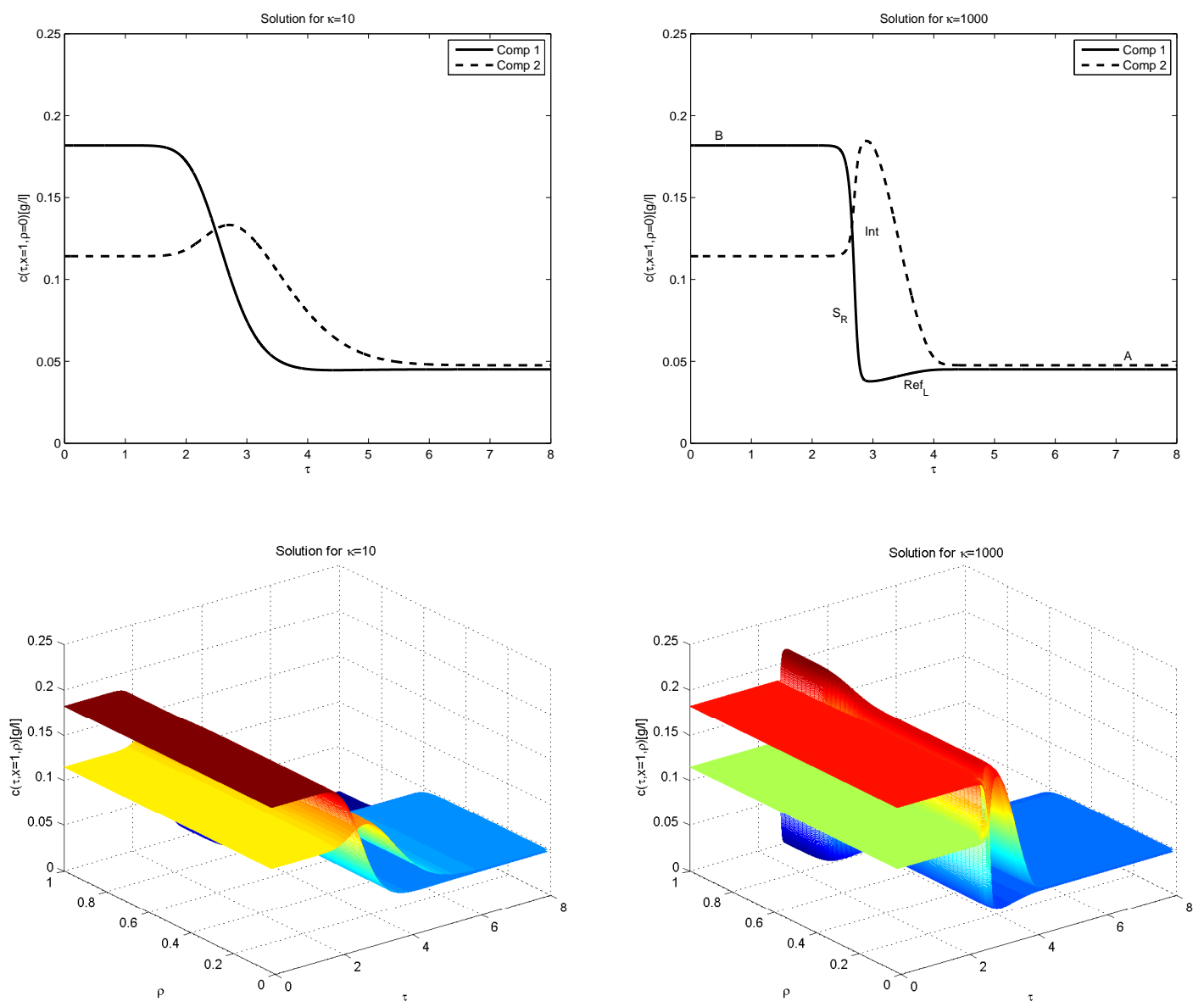

Figure 5: Plots of Simulation 1 of Case 1 at the column outlet using $100 \times 50$ grid points. 

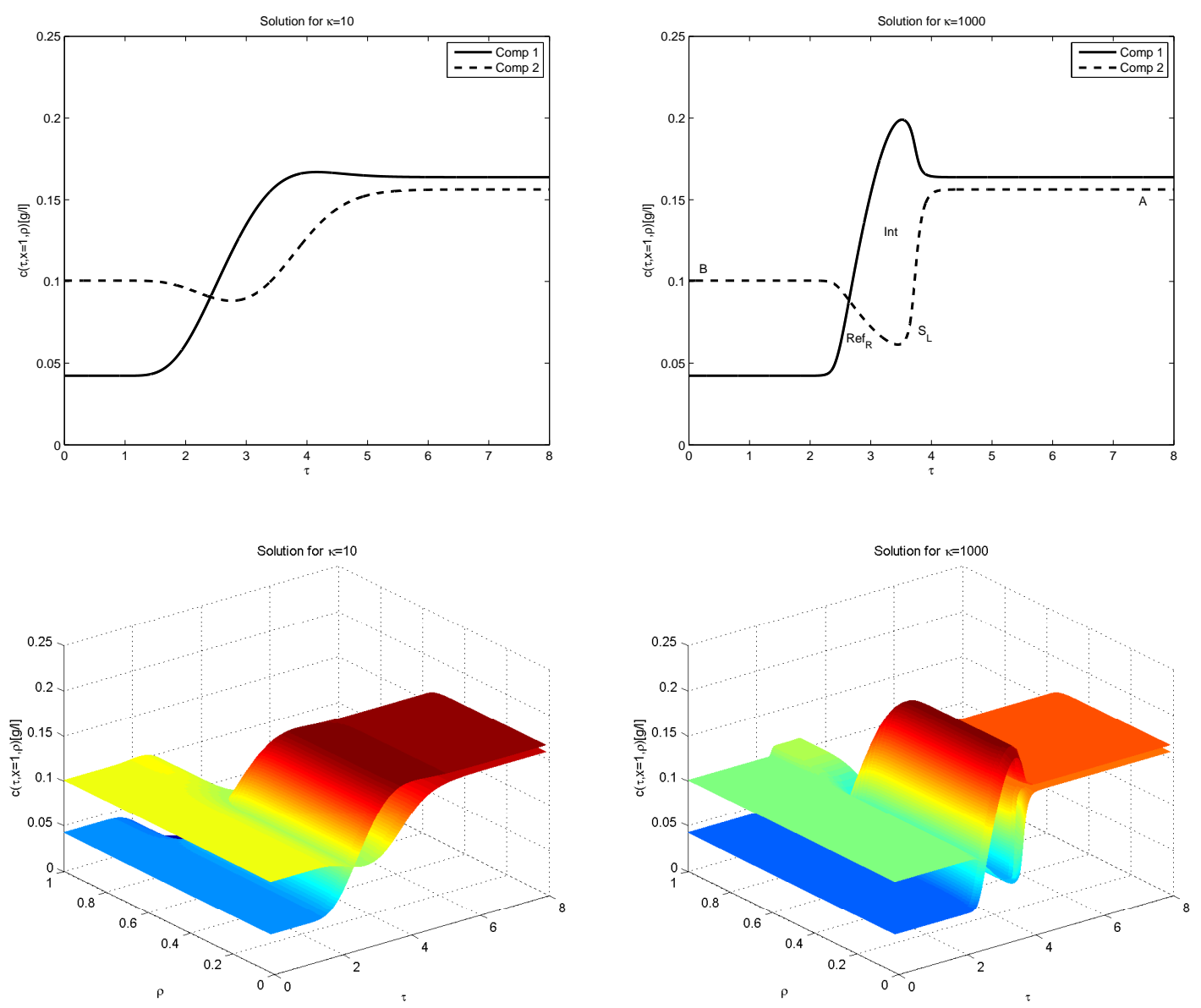

Figure 6: Plots of Simulation 2 of Case 1 at the column outlet using $100 \times 50$ grid points. 

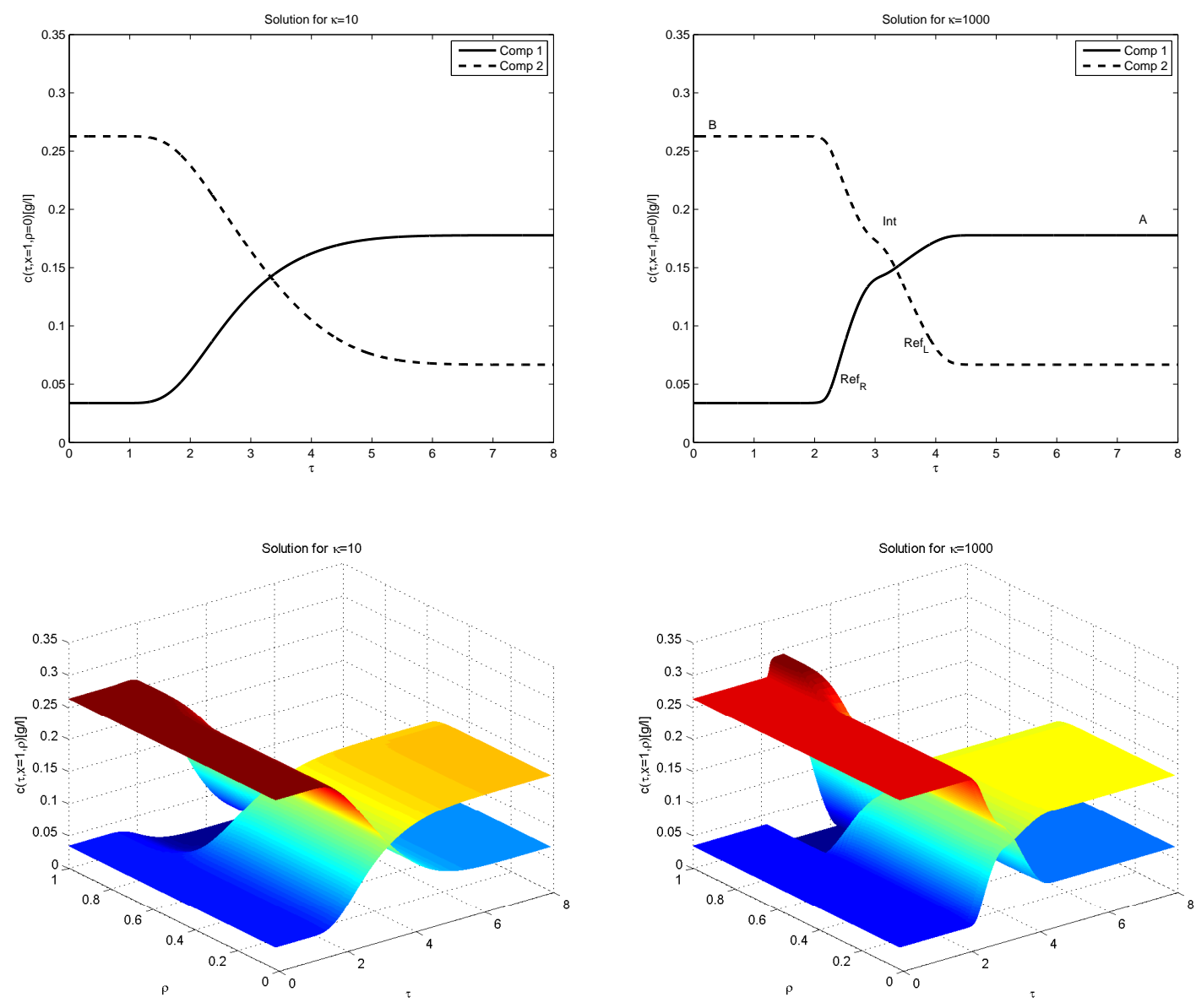

Figure 7: Plots of Simulation 3 of Case 1 at the column outlet using $100 \times 50$ grid points. 

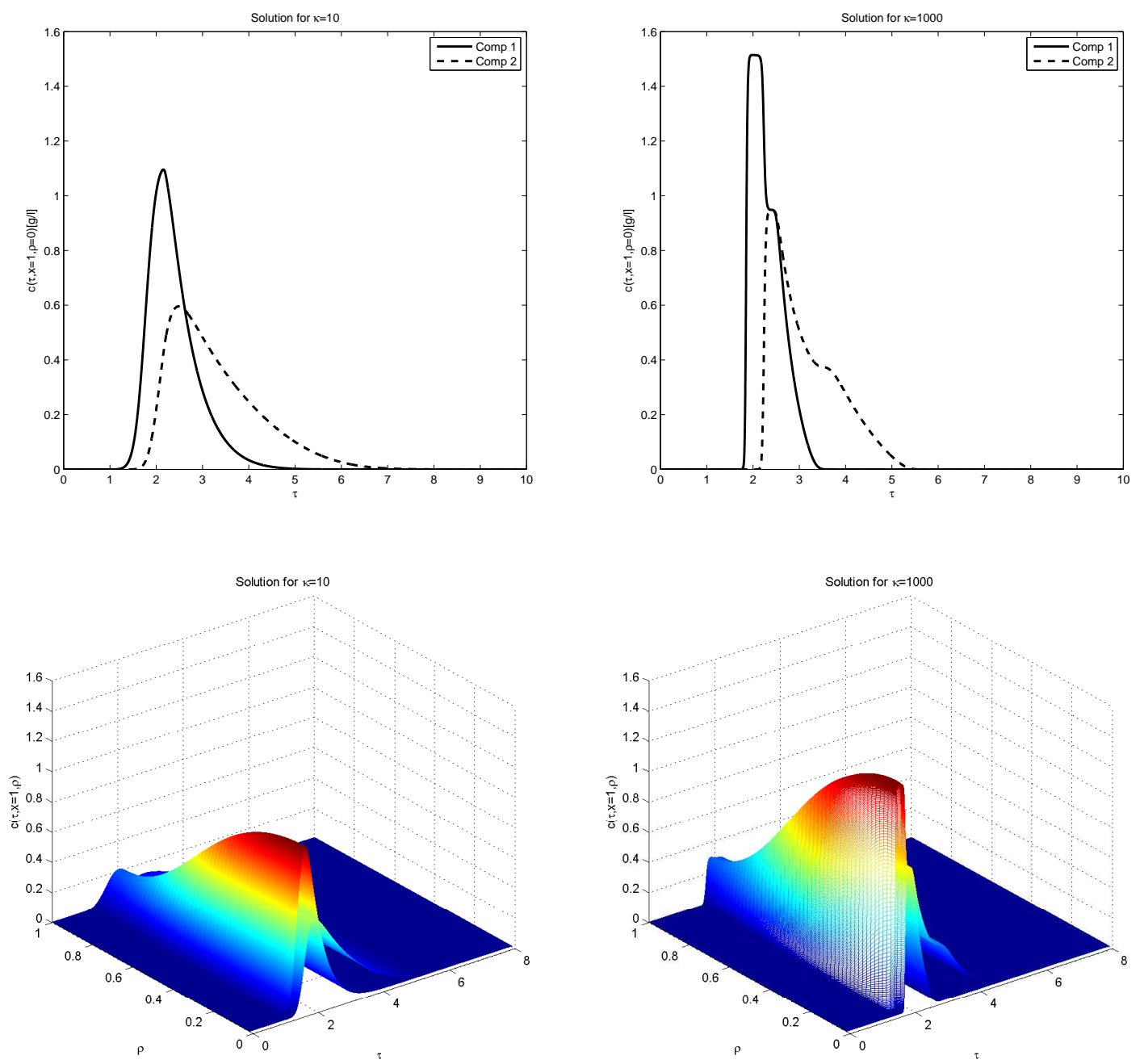

Figure 8: Two-component nonlinear elution profiles at the column outlet using $100 \times 50$ grid points. 

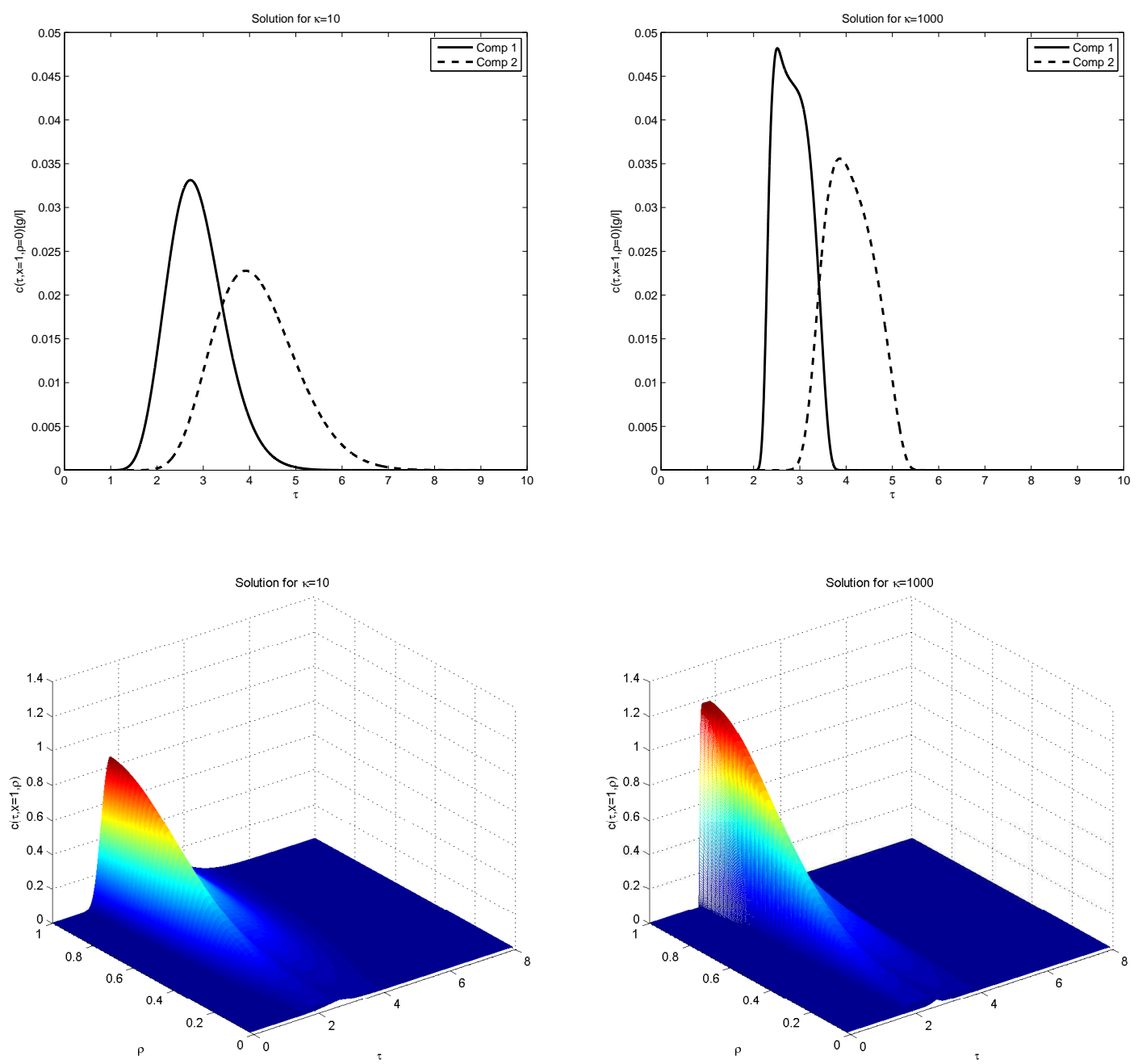

Figure 9: Two-component nonlinear elution profiles at the column outlet using $100 \times 50$ grid points. 

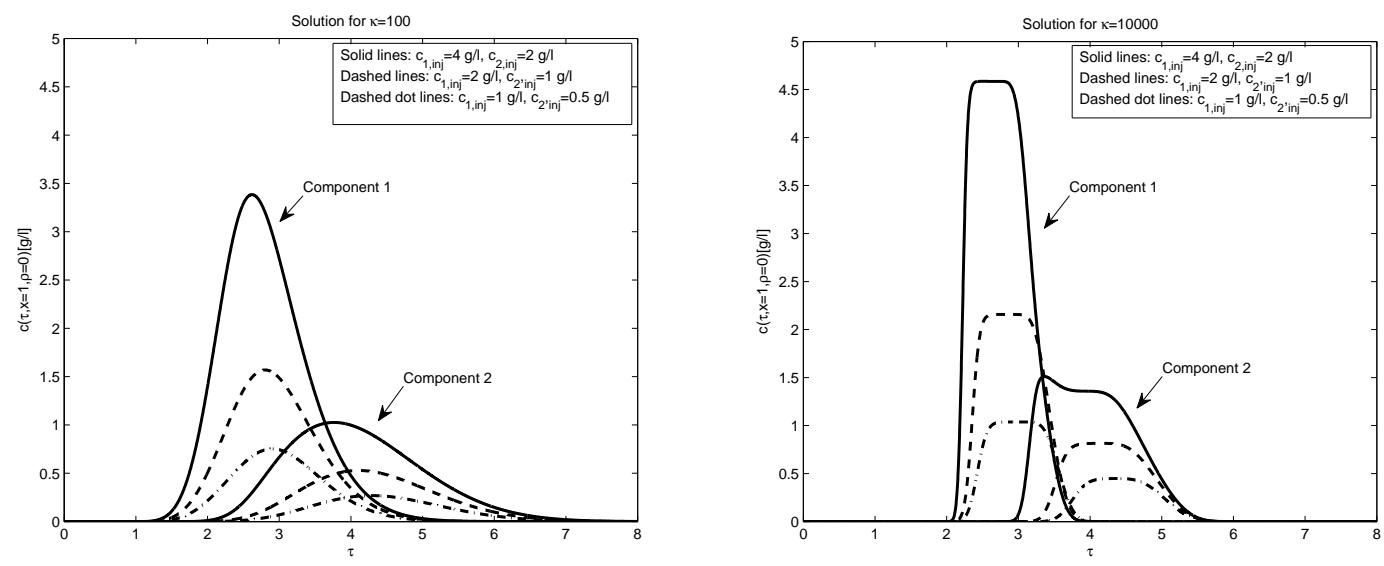

Figure 10: Two-component nonlinear elution profile at the column outlet using different injection volumes (a): For $\kappa=10^{2}$, (b): For $\kappa=10^{4}$.

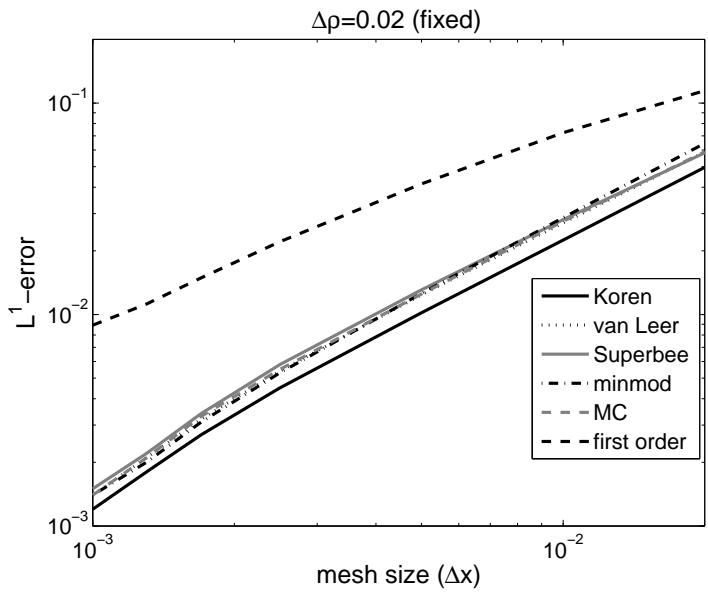

Figure 11: $L^{1}$-Errors for component 1 in the two-component nonlinear elution. 

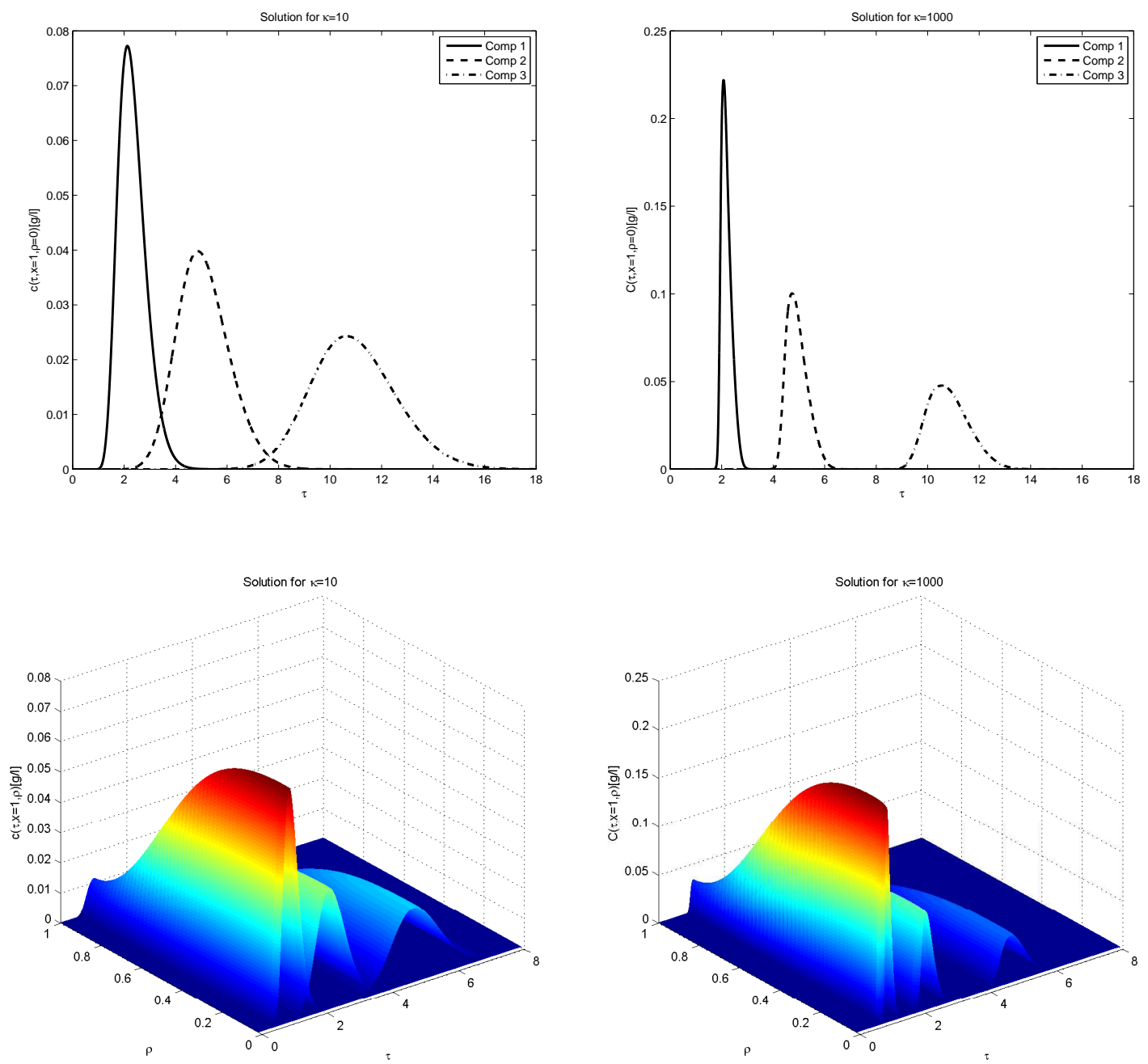

Figure 12: Three-components nonlinear elution profiles at the column outlet using $50 \times 50$ grid points. 